Advance Access publication 2018 November 21

GJI Geomagnetism, rock magnetism and palaeomagnetism

\title{
Polar caps during geomagnetic polarity reversals
}

\author{
Bruno Zossi, ${ }^{1,2}$ Mariano Fagre, ${ }^{2,3}$ Hagay Amit ${ }^{4}$ and Ana G. Elias ${ }^{\oplus 1,2}$ \\ ${ }^{1}$ INFINOA (CONICET-UNT) \& Laboratorio de Física de la Atmosfera, Dpto. de Física, Facultad de Ciencias Exactas y Tecnología, Universidad Nacional de \\ Tucuman, Tucuman 4000,Argentina.E-mail: aelias@herrera.unt.edu.ar \\ ${ }^{2}$ Consejo Nacional de Investigaciones Cientificas y Técnicas, CONICET, Godoy Cruz 2290, Argentina \\ ${ }^{3}$ Laboratorio de Telecomunicaciones, Dpto. de Electricidad, Electrónica y Computación, Facultad de Ciencias Exactas y Tecnología, Universidad Nacional \\ de Tucuman, Tucuman 4000, Argentina \\ ${ }^{4}$ CNRS, Université de Nantes, Nantes Atlantiques Universités, Laboratoire de Planétologie et de Géodynamique, Nantes 44322, France
}

Accepted 2018 November 20. Received 2018 October 16; in original form 2018 July 31

\begin{abstract}
SUMMAR Y
Changes in the Earth's magnetic field can deeply modify the polar caps and auroral zones, which are the regions of most frequent precipitation of energetic particles. The present field is characterized by a dominant dipole plus weaker multipolar components. The field varies greatly in time, with the most drastic changes being polarity reversals that take place on average every $\sim 200000$ yr. During a polarity transition the field magnitude may diminish to about 10 per cent of its value prior to the reversal due to a decreasing dipolar component and by becoming mostly multipolar in nature. Polar caps depend on the geomagnetic field configuration so changes in their morphology are expected as a consequence of the variation and reversal of this field. We model polar caps' location by considering a superposition of the internal geomagnetic field and a uniform external field and then following the open field lines to the Earth's surface. Polar caps' location and shape for different magnetic field reversal scenarios are analysed in this work. Two polar caps near the present dipole axis intersection with the Earth's surface prevail for a dipole decrease to a certain extent, below which the southern hemisphere polar cap moves to mid-latitudes. An axial dipole collapse gives a pair of polar caps both at mid-latitudes of the southern hemisphere, while in a dipole rotation scenario the polar caps reside at the equator. If reversals occur due to an energy cascade from the dipole to higher degrees, more than two polar caps may appear. In our energy cascade scenario, four polar caps at various latitudes of both hemispheres prevail. These results indicate that during reversals auroral zones may reach mid- and low-latitude regions, and the atmosphere may become more vulnerable to the direct effect of energetic particle precipitation. This vulnerability is particularly striking at the southern hemisphere where reversed flux patches appear on the core-mantle boundary and weak intensity characterizes the present field at the Earth's surface.
\end{abstract}

Key words: Environmental magnetism; Magnetostratigraphy; Palaeointensity; Palaeomagnetism; Reversals: process, time scale, magnetostratigraphy.

\section{INTRODUCTION}

The Earth's magnetic field shields the planet from the solar wind. However, some energetic particles precipitate along geomagnetic field lines through the magnetospheric plasma sheet into the auroral ionosphere powering current systems, heating the upper atmosphere and displaying auroras (Russell 2000; Hunsucker \& Hargreaves 2003). Polar caps are these regions above the Earth's surface of open magnetic field lines. Their boundary, also called open-closed field line boundary, separates the open magnetic field lines connected to the interplanetary magnetic field from the closed lines of the magnetosphere.
The magnetosphere varies over different timescales. On an hourly to daily scale its variations are due to changes in the solar wind conditions, while on long-term timescales, much greater than decadal, they are linked to changes in the Earth's magnetic field that affect the size as well as the geometry of the magnetosphere (Vogt et al. 2007). In the present-day Earth's magnetosphere, the dipole axis is almost perpendicular to the solar wind flow direction. Assuming that magnetospheric configuration varies self-similarly with the dipole moment intensity $M$, several authors derived scaling relations for the location of its boundary which are valid for pure dipolar fields (Siscoe \& Chen 1975; Vogt \& Glassmeier 2000; Glassmeier et al. 2004). Willis et al. (2000) assumed a fixed spherical magnetopause 
that behaves as a perfectly conducting surface for an idealized possible multipolar configuration during a geomagnetic reversal.

Polar caps' location and shape can be determined by identifying and following these open field lines using mathematical modelling that can be executed with different complexity levels. On one extreme numerical magnetohydrodynamic (MHD) models consider every detail of the dynamic structure and interaction between the solar wind and the magnetosphere (Gombosi et al. 2004). On the other extreme oversimplified fast models can be easily executed and manipulated (Polvani et al. 2017). The latter was used in this work. Even though Polvani et al. (2017) refer to climate models, their arguments can be well applied to our case. They refer to the Earth system models that are usually extremely complex. To overcome this complexity, the Community Earth System Model, which is a fully coupled global climate model maintained by the National Center for Atmospheric Research, will include a hierarchy of simpler models, which can shed some light and help to obtain a fundamental understanding of the studied system, which is often not accessible even with extremely complex simulations, precisely because of their complexity.

The Earth's magnetic field at present can be approximated by a geocentric dipole with its axis tilted about $11^{\circ}$ with respect to the Earth's rotation axis. This dipole accounts for $\sim 80$ per cent of the magnetic field at the Earth's surface and the remaining $\sim 20$ per cent is made up by non-dipolar components (Merrill et al. 1998). Palaeomagnetic measurements show that the Earth's magnetic field varies greatly, with the most drastic changes being polarity reversals that take place on average every $\sim 200000 \mathrm{yr}$ considering the last 80 million yr. The duration of the transition period leading to a polarity reversal is $\sim 1000-8000 \mathrm{yr}$ (Clement 2004).

Although the understanding of geomagnetic reversals has improved considerably over the years with palaeomagnetic studies and geodynamo simulations (Jacobs 1994; Valet et al. 2012; Valet \& Fournier 2016), some properties are still under debate. Hulot et al. (2010) found based on numerical dynamos that the chaotic nature of the geodynamo renders predicting when the next magnetic field reversal will occur impossible. Morzfeld et al. (2017), using low-dimensional models, predicted that the field will not reverse in the next $4000 \mathrm{yr}$. There is also the uncertainty about the dominance of a dipolar or multipolar configuration during a polarity transition. Obviously the axial dipole vanishes during a reversal when the dipole axis crosses the equator. Amit et al. (2010) summarized several reversal scenarios with two extremes for the dipolar component: a dipole collapse and a dipole rotation from one hemisphere to the other. In the latter case, only the axial dipole would vanish by transferring its energy to the equatorial components. Regarding the non-dipolar field, Amit et al. (2010) considered three main possibilities: (1) decrease and recovery in phase with the dipole collapse, (2) remains unchanged or (3) grows throughout the reversal possibly due to energy transfer from the dipole (Amit \& Olson 2010; Huguet \& Amit 2012) or dynamo configurations favouring the generation of a non-dipolar field.

If during a polarity transition either a multipolar configuration or an equatorial dipole prevails, a significant change in magnetic poles number and location is expected. Changes in the geomagnetic field can then deeply alter the zones of particle precipitation into the upper atmosphere, altering also the spatial structure of neutral temperature and winds, ionosphere electron density, chemical processes and ionosphere-magnetosphere coupling.

Siscoe \& Chen (1975) were among the first to study in detail the effects of a polarity transition on the Earth's magnetosphere.
They deduced that the stand-off distance $R_{\mathrm{MP}}$ of the dayside magnetopause scales with the dipole magnetic moment, $M$, as $R_{\mathrm{MP}} \propto M^{1 / 3}$ and that the latitude of the edge of the polar cap, $\lambda_{\mathrm{p}}$, varies in terms of $M$ according to $\cos \left(\lambda_{\mathrm{p}}\right) \propto(1 / M)^{1 / 6}$, that is, it moves equatowards as $M$ decreases. Vogt \& Glassmeier (2001) and Glassmeier et al. (2004) derived a similar scaling law given by $\cos \left(\lambda_{\mathrm{p}}\right) \propto(1 / M)^{\gamma-1 / 2}$, where $\gamma$ is the scaling exponent of the tail radius, $R_{\mathrm{T}}$, that is, $R_{\mathrm{T}} \propto M^{\gamma}$. Siscoe $\&$ Sibeck (1980) estimated the shape of the auroral zones during a polarity reversal with a field dominated by non-dipolar components. They found a geographical displacement and an elongation of the polar cap borders, enclosing an area which remained roughly constant. Sterenborg et al. (2011) considered a number of solar wind scenarios $\sim 3.5$ Gyr ago to estimate the polar cap latitude extent for different Earth's reversal conditions. However, they used only the dipolar field component.

Directly linked to polar caps are the auroral zones and ovals, since polar cap boundaries also correspond to their inner limit. This limit becomes enlarged under geomagnetic storm conditions. The auroral oval is a band of almost permanent auroras about $10^{\circ}$ wide in latitude, where particles from the Sun are accelerated down the magnetic field lines onto the upper atmosphere. It is broader on the nightside of the Earth and is more or less fixed in space while the Earth rotates underneath it. It is sometimes used as a synonym of auroral zone, but there are some differences (Akasofu, 1983; Feldstein, 2016). The auroral zone corresponds only to the nightside part of the oval, and the field lines here are closed and map to the plasma sheet in the magnetosphere. For the present day field, the belt of maximum occurrence of auroras in terms of the number of nights of auroral appearance per year is called auroral zone. It is a narrow, circular belt with its centre line at $67^{\circ}$ geomagnetic latitude. However, the instantaneous belt of aurora, which is called auroral oval, is eccentric with respect to the geomagnetic pole and lies at around $67^{\circ}$ at the midnight meridian and $76^{\circ}$ at the noon meridian. The oval is fixed with respect to the Sun and the Earth rotates under this oval. The auroral zone is the locus of the midnight part of the average auroral oval, as the Earth rotates under the oval once a day (Akasofu, 1983).

In this work, the polar caps' location and shape are determined for different geomagnetic field configurations during reversals. These scenarios were recently considered by Zossi et al. (2018) in a study of the ionospheric Hall and Pedersen conductances. The method used to estimate the polar caps is described in Section 2, followed by the obtained results in Section 3. A discussion is given in Section 4 and the main conclusions are summarized in Section 5.

\section{METHODOLOGY}

The different geomagnetic field configurations are described in subsection 2.1. We consider a superposition of the internal geomagnetic field plus a uniform external field as outlined in subsection 2.2 to obtain the magnetosphere configuration. Polar caps were assessed by following the resulting open field lines and tracing them to the Earth's surface as explained in subsection 2.3.

\subsection{The Earth's magnetic field configurations}

The International Geomagnetic Reference Field (IGRF version 12) model (Thébault et al. 2015) was used to specify the initial (before the reversal) magnetic field $\mathbf{B}$ for all scenarios. $\mathbf{B}$ is given in terms of the internal magnetic scalar potential $V$ by $\mathbf{B}=-\nabla V$, which is 
expanded by the series

$$
\begin{aligned}
V(r, \theta, \varphi, t)= & a \sum_{n=1}^{13} \sum_{m=0}^{n}\left(\frac{a}{r}\right)^{n+1} \\
& \times\left\{\left[g_{n}^{m}(t) \cos (m \varphi)+h_{n}^{m}(t) \sin (m \varphi)\right] P_{n}^{m}(\cos \theta)\right\},
\end{aligned}
$$

where $a=6371.2 \mathrm{~km}$ is the Earth's mean reference spherical radius, $r$ the radial distance from the centre of the Earth, $\theta$ the geocentric co-latitude, $\varphi$ the east longitude, $P_{n}^{m}(\cos \theta)$ the Schmidt quasi-normalized associated Legendre functions of degree $n$ and order $m$, and $g_{n}^{m}$ and $h_{n}^{m}$ the Gauss coefficients which are functions of time $t$.

We considered the first three degrees only, that is, $n=1,2$ and 3, which correspond to the dipolar, quadrupolar and octupolar components, respectively, because multipolar components decrease with $r^{-(n+1)}$, that is, very rapidly with radial distance. The transitional field was first modelled by gradually decreasing the coefficients of the dipolar component, that is, those corresponding to $n=1\left(g_{1}^{0}, g_{1}^{1}\right.$ and $\left.h_{1}^{1}\right)$, while keeping unchanged the quadrupolar and octupolar coefficients. The three other end-member scenarios considered for the reversal are: an axial dipole collapse where the axial dipolar component is set to zero while maintaining the equatorial dipole components as well as degrees 2 and 3 unchanged (i.e. only setting $g_{1}^{0}=0$ ), a dipole rotation where the energy of the axial component is transferred to the equatorial components proportional to their original energies (i.e. setting $g_{1}^{0}=0$ and increasing $g_{1}^{1}$ and $h_{1}^{1}$ ), and a third scenario consisting of an energy cascade where the energy of the dipolar components is transferred to the quadrupolar and octupolar components also proportional to the original energy of each degree and order (i.e. setting $g_{1}^{0}, g_{1}^{1}$ and $h_{1}^{1}$ to zero and increasing the next 12 Gauss coefficients, which correspond to the 5 quadrupolar and the 7 octupolar terms). For the last two scenarios, a constant total magnetic energy on the core-mantle boundary calculated based on the Mauersberger-Lowes spectrum (Lowes 1974) was considered, given by

$R=\sum_{n} R_{n}=\sum_{n}(n+1)\left(\frac{a}{c}\right)^{2 n+4} \sum_{m}\left[\left(g_{n}^{m}\right)^{2}+\left(h_{n}^{m}\right)^{2}\right]$,

where $c=3480 \mathrm{~km}$ is the radius of the core. The configuration of the remaining components in each case (the equatorial dipole components in the first, and the quadrupolar and octupolar components in the second) was maintained by keeping the proportions constant. That is, given $g_{1}^{0}, g_{1}^{1}$ and $h_{1}^{1}$ for present conditions, the dipole rotation reversal scenario consists of $g_{1}^{* 0}=0$ and $g_{1}^{* 1}$ and $h_{1}^{* 1}$ given by

$$
\begin{aligned}
\frac{2\left(\frac{a}{c}\right)^{6}\left(g_{1}^{* 1}\right)^{2}}{R_{1}} & =\frac{2\left(\frac{a}{c}\right)^{6}\left(g_{1}^{1}\right)^{2}}{R_{1}-2\left(\frac{a}{c}\right)^{6}\left(g_{1}^{0}\right)^{2}} \\
\frac{2\left(\frac{a}{c}\right)^{6}\left(h_{1}^{* 1}\right)^{2}}{R_{1}} & =\frac{2\left(\frac{a}{c}\right)^{6}\left(h_{1}^{1}\right)^{2}}{R_{1}-2\left(\frac{a}{c}\right)^{6}\left(g_{1}^{0}\right)^{2}},
\end{aligned}
$$

where $R_{1}=R_{1}^{*}$, that is, $\left(g_{1}^{0}\right)^{2}+\left(g_{1}^{1}\right)^{2}+\left(h_{1}^{1}\right)^{2}=\left(g_{1}^{* 1}\right)^{2}+\left(h_{1}^{* 1}\right)^{2}$. For the energy cascade scenario, the transfer to the quadrupole and octupole coefficients considering $g_{1}^{* 0}=g_{1}^{* 1}=h_{1}^{* 1}=0$ is given by

$$
\begin{aligned}
& \frac{(n+1)\left(\frac{a}{c}\right)^{2 n+4}\left(g_{n}^{* m}\right)^{2}}{R}=\frac{(n+1)\left(\frac{a}{c}\right)^{2 n+4}\left(g_{n}^{m}\right)^{2}}{R_{2}+R_{3}} \\
& \frac{(n+1)\left(\frac{a}{c}\right)^{2 n+4}\left(h_{n}^{* m}\right)^{2}}{R}=\frac{(n+1)\left(\frac{a}{c}\right)^{2 n+4}\left(h_{n}^{m}\right)^{2}}{R_{2}+R_{3}} .
\end{aligned}
$$

Here $R=R_{2}^{*}+R_{3}^{*}$, since $R_{1}^{*}=0$.

Note that the reversal scenarios described above are just simple selected cases that may illustrate possible transitional fields. Although the size of the Gauss coefficient of each spherical harmonic degree and order varies in at least one of these scenarios, clearly these four scenarios do not cover all possible transitional fields. For example, in all scenarios including energy cascade the relative strengths of the quadrupole and octupole terms remain unchanged, while in reality the transitional geomagnetic field may be more complex.

\subsection{Magnetic field superposition}

A simple configuration of the magnetosphere and the open field lines can be obtained by the superposition of a dipole field, for the present Earth's field conditions, representing the internal geomagnetic field of the core origin, and a uniform field in the direction of the interplanetary external field (Stern 1973, Hill \& Rassbach 1975). The polar cap surfaces are obtained by projecting the open magnetic field lines of the resulting configuration to the Earth's surface.

In general, the total field, $B_{\mathrm{T}}$, of the superposition between a uniform field of intensity $B_{\mathrm{U}}$ inclined an angle $\alpha$ with respect to the geographic North (taken as the reference for the polar angle $\theta$ ), and a field $\mathbf{B}$ of spherical components $B_{\mathrm{r}}, B_{\theta}$ and $B_{\varphi}$, results:

$B_{\mathrm{rT}}=B_{\mathrm{U}} \cos (\theta-\alpha)+B_{\mathrm{r}}$

$B_{\theta \mathrm{T}}=-B_{\mathrm{U}} \sin (\theta-\alpha)+B_{\theta}$

$B_{\varphi \mathrm{T}}=B_{\varphi}$.

For a given $\mathbf{B}$ the polar caps will clearly depend on $\alpha$. In the ideal case of an axial dipolar field, the superposition is with a field that is parallel to the dipole field in the equatorial plane $(\alpha=0)$, hence the only open field line would be that with $90^{\circ}$ inclination, that is, the polar caps are reduced to a point located at each pole. For the opposite condition, that is, $\alpha=180^{\circ}$, the open field lines projected to the Earth's surface delineate a circular polar cap with the maximum possible area for given $B_{\mathrm{U}}$ and $\mathbf{B}$. These situations are clearly depicted in fig. 1 of Hill \& Rassbach (1975). For a general multipolar configuration, a sweep for $\alpha$ between $0^{\circ}$ and $180^{\circ}$ should be considered in order to obtain the polar caps' configurations. We considered $B_{\mathrm{U}}=49 \mathrm{nT}$ that is almost 10 times greater than typical quiet interplanetary magnetic field, IMF, values since it represents the equivalent magnetic field that would produce the solar wind dynamic pressure at the subsolar point converted into magnetic pressure (Hill \& Rassbach 1975). This value results from considering (Beard 1960)

$$
\left(2 \rho v^{2}\right)_{\mathrm{sw}}=\frac{B^{2}}{2 \mu_{0}},
$$

where $\rho$ is the proton density (proton mass multiplied by the solar wind numerical proton density), $v$ the solar wind velocity, $\mu_{\mathrm{o}}$ the magnetic permeability and the subscript 'sw' denotes 'solar wind'. Mean solar wind values for quiet conditions used for $\rho$ and $v$, namely $\rho=2.5 \times 10^{6} \mathrm{~m}^{-3}$ and $v=350 \mathrm{~km} \mathrm{~s}^{-1}$, yield $|\mathbf{B}|=49 \mathrm{nT}$, which is used as $B_{\mathrm{U}}$.

\subsection{Magnetic field lines estimation}

Following magnetic field lines is not a trivial mathematical problem. In the case of symmetrical fields, there are in general analytical solutions for the field lines. For generic fields numerical simulations can 
be performed with a computer algebra system such as Mathematica (Hastings et al. 2016).

If the magnetic field $\mathbf{B}=\left(B_{x}, B_{y}, B_{z}\right)$ is known as a function of position, then the magnetic field line element $\mathbf{d l}=(\mathrm{d} x, \mathrm{~d} y, \mathrm{~d} z)$ can be obtained using the condition that the line direction at every point is the direction of the field at that point, that is, $\mathbf{d l} \times \mathbf{B}=0$. Hence the magnetic field line is defined by

$\frac{\mathrm{d} x}{B_{x}}=\frac{\mathrm{d} y}{B_{y}}=\frac{\mathrm{d} z}{B_{z}}$.

Considering sufficiently small differential elements $\mathrm{d} x, \mathrm{~d} y$ and $\mathrm{d} z$, it is possible to plot the magnetic field lines by solving eq. (7) with a fourth-order Runge-Kutta method. Field line visualizations are extremely useful in imaging solutions of numerical dynamo simulations, including during polarity reversals (Aubert et al. 2008).

\section{RESULTS}

Fig. 1 shows the Earth's magnetic field intensity and inclination for a dipole collapse scenario: the current configuration and two stages where the dipolar components were decreased by 50 and 90 per cent, respectively, of their current values. The patterns of intensity and inclination are only mildly modified by a 50 per cent reduction, in which the dipole still remains the dominant degree. In this case and in the current field morphology, the intensities are lowest at the South Atlantic (Figs 1a and c; see also, e.g. Terra-Nova et al. 2017) and the absolute values of the inclination are larger at higher latitudes (Figs 1b and d). However, when the dipole is reduced to 10 per cent the higher degrees take over, resulting in distinctive more complex patterns of intensity and inclination (Figs 1e and f). Fig. 2 shows the three other end-member scenarios for the reversal: the axial dipole collapse, the dipole rotation and the energy cascade. Note that the axial dipole collapse scenario (Figs 2a and b) gives very different results from the final stage of dipole collapse, in which 10 per cent of the dipole is retained (Figs 1e and f). For example, lowest intensities appear in the former in the western hemisphere whereas in the latter in the northern hemisphere. The dipole rotation scenario yields a clear equatorial dipole signature in the inclination at the height of a reversal (Fig. 2d), as expected. The energy cascade scenario (Figs 2e and f) produces similar complex patterns as in the final stage of the dipole collapse scenario (Figs 1e and f).

Fig. 3 shows, as an example of the method, the magnetic field lines obtained with eq. (5) summing the internal geomagnetic field configurations considered in Figs. 1-2 and a uniform external field of $49 \mathrm{nT}$ intensity. The dominant axisymmetry of the field is lost when the axial dipole vanishes; this can be noted from the difference between Figs 3(c) and (e) compared to Figs 3(b) and (d), respectively, which show different meridional cross-sections of the same magnetic field configurations.

As expected, far from the Earth the imposed uniform external field dominates the overall configuration (downward-pointing field lines in Fig. 3) while closer to the Earth the internal geomagnetic field dictates the orientation. In the present day configuration close to the Earth, the magnetic field lines are organized around two magnetic poles near where the dipole axis intersects the surface (Fig. 3a). Axial dipole collapse in the $90^{\circ}$ meridian cross-section also gives two magnetic poles but near the equator (Fig. 3b). In other cases the multipolar configurations result in four magnetic poles (Figs 3c, d and e).

Polar caps were determined using eq. (7) for open field lines connecting to the uniform external field, given by eq. (5). Figs 4 and 5 show the polar caps obtained for the different geomagnetic field scenarios considered.

In the case of a gradual decrease of the dipolar component, since the multipolar field decreases with altitude faster than the dipole field, the latter is still dominant in the solar wind coupling processes at the magnetosphere boundary. This can be noted in Fig. 4 where only two polar caps appear near where the dipole axis intersects the Earth's surface for a 50 per cent residual dipolar component. Even for a 10 per cent residual dipolar component only two polar caps appear but here the southern polar cap is off the dipole axis to mid-latitudes. This means that at the magnetopause position the dipolar field still dominates the interaction with the solar wind. However, in these cases polar caps do not behave according to the scaling laws mentioned previously, according to which polar cap limits should extend equatorwards with a consequent increasing polar cap area. In fact, the scaling relation does not hold in these cases where self-similarity is almost completely lost. Instead, the polar cap elongates and its centre shifts equatorwards as in Fig. 4(c) in the southern hemisphere. This happens because open field lines at the magnetopause are dominated by the dipolar component, but when approaching the Earth the field configuration begins to be dominated by multipolar components, which redirect and compress the area where the fields reach the Earth. Polar caps appear where the intensity and the absolute inclination are large. For present day and 50 per cent reduction this gives two polar cap regions $180^{\circ}$ apart. For 90 per cent reduction the polar caps appear at Siberia and south of Australia (Fig. 4c) where large intensities (Fig. 1e) and large absolute inclinations (Fig. 1f) are found.

In the cases of axial dipole collapse and dipole rotation, a clear displacement of the two polar caps to low latitudes can be noted as a result of the dominant role of the equatorial dipole component (Figs 5a and b). However, while in the dipole rotation scenario the polar caps reside at the equator due to the dominance of the enhanced equatorial dipole (Fig. 5b), in the axial dipole collapse the polar caps are almost exclusively in the southern hemisphere (Fig. 5a). This is due to the overall higher intensities in the southern hemisphere in this scenario (Fig. 2a) combined with the particular longitudes where large absolute inclinations appear for the same scenario (Fig. $2 b)$. In the energy cascade case, due to a non-axial quadrupolar dominance, four polar caps are found (Fig. 5c). Once again note that these four polar caps correspond remarkably well to locations with large intensities (Fig. 2e) and large absolute inclinations (Fig. $2 \mathrm{f})$. The octupolar field is less influential because it decreases with altitude faster than the quadrupolar field.

\section{DISCUSSION}

\subsection{Comparison with MHD models}

In this paper polar caps were determined using a field superposition and field-lines tracing method, which is an intuitive way to calculate polar caps' location. Even though we considered geomagnetic fields expanded until spherical harmonic degree and order 3 only, inclusion of higher degrees does not change our results. Of course the approach can be improved by adding realistic magnetopause, currents and magnetotail. However, our simple approach based on a reasonable approximation for the magnetospheric field benefits from a low computational cost without resorting to neither big databases nor large MHD simulations.

Zieger et al. (2006) considered a pure axial dipole moment. They observed that the scaling exponent of the power-law relation between the polar cap latitude and the dipole moment depends also 


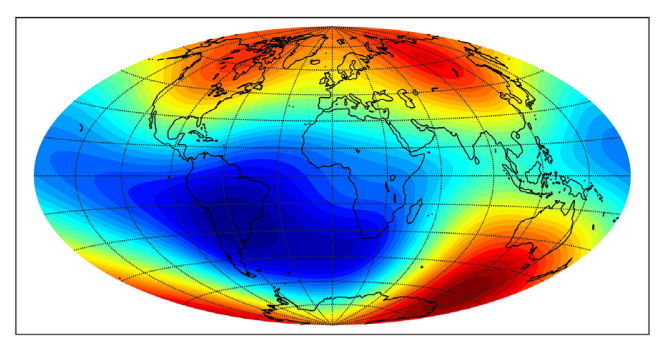

(a)

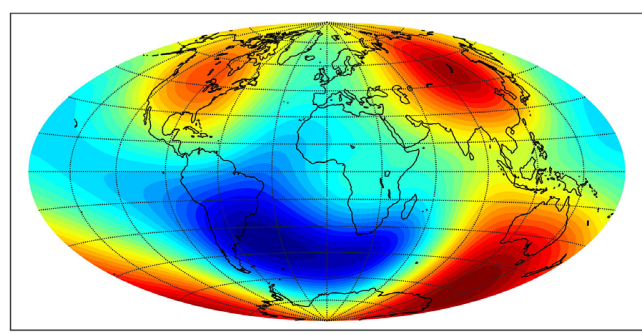

(c)
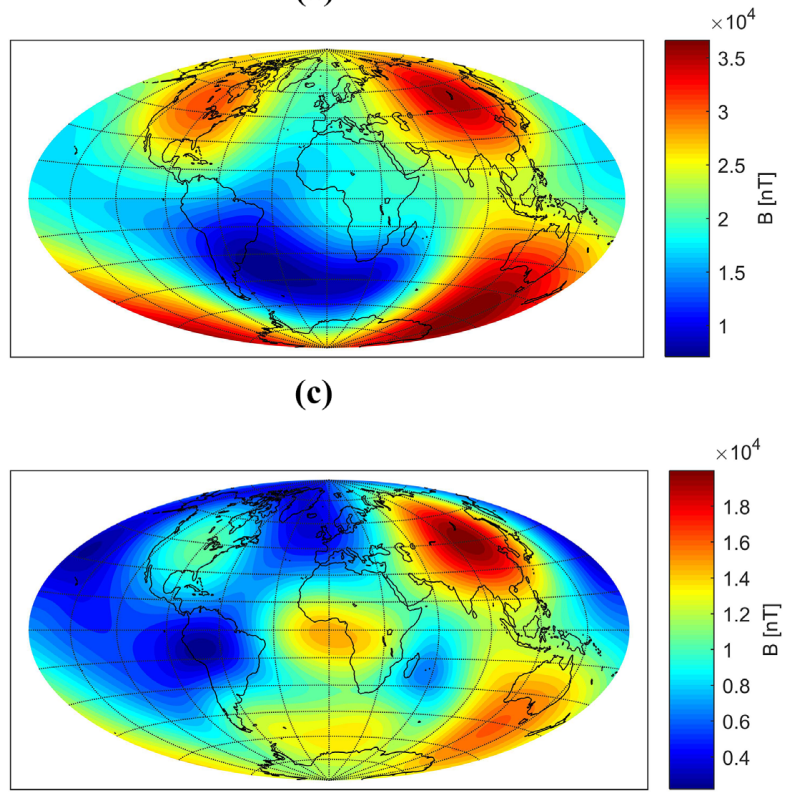
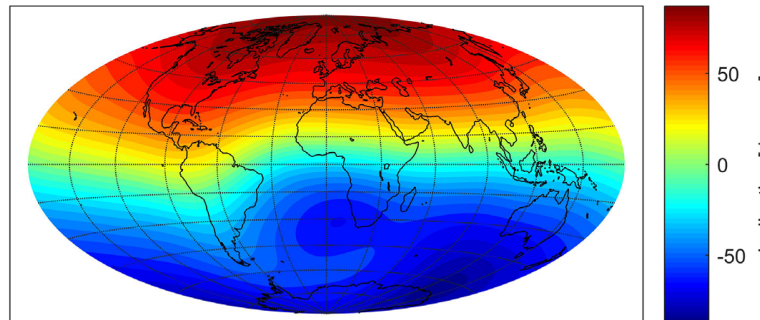

(b)
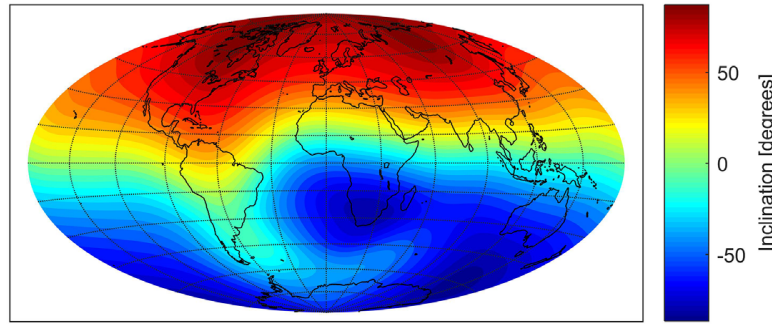

(d)

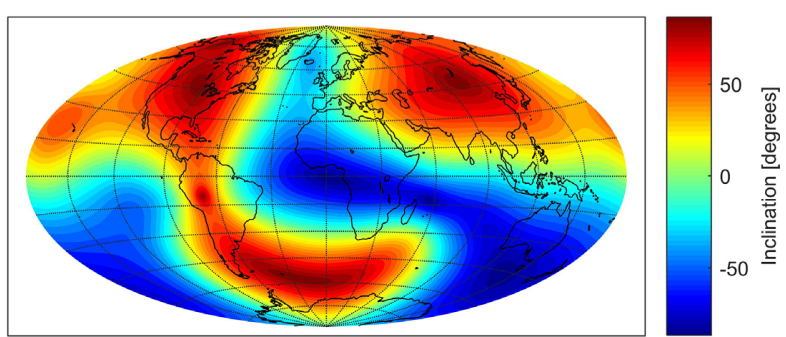

(f)

Figure 1. Intensity of the Earth's magnetic field $|\mathbf{B}|$ (nT) and inclination I (degrees) obtained from IGRF-12 for (a and b) present conditions, (c and d) 50 per cent decrease of dipolar components and (e and f) 90 per cent decrease of dipolar components. Note the different scales for intensity.

on the IMF axial component $B_{z}$. For strong negative $B_{z}$ the polar cap size increases faster with the decreasing dipole moment than in the self-similar model. Our results are in good agreement with their results for $B_{z}=0$. The scale exponent of the polar cap size is the ideal $1 / 6$ in both cases.

In the case of the dipole rotation scenario (Fig. 5b), as Zieger et al. (2004) pointed out in their equatorial dipolar magnetosphere, the open field line configuration will change during daily rotation of the Earth. Fig. 5(b) corresponds to the superposition of the equatorial dipole parallel and antiparallel to the solar wind flow direction.

Vogt et al. (2007) considered a dipole plus an axial quadrupole geomagnetic field. They pointed out that at one of the geomagnetic poles, the quadrupole and the dipole fields point into opposite directions leading to a decrease in the total field strength, so the open solar wind flux has to be distributed over a larger surface area. At the opposite pole, where both field contributions point to the same direction, the total field strength increases resulting in a smaller polar cap. In the present-day geomagnetic field in terms of total quadrupolar energy on the core-mantle boundary, the axial, tesseral and sectoral components represent 23, 65 and 12 per cent, respectively. In our reversal scenarios we keep these proportions constant, so the axial quadrupole is not dominant. Consequently, instead of an increasing polar cap in one hemisphere and a decreasing in the other, we find an elongation of almost circular initial polar caps and also a shift of the centre location of the southern polar cap as the axial dipolar component decreases (Fig. 4).

\subsection{Scaling laws}

The scaling relations proposed by previous studies, which are rigorously valid under the hypothesis of self-similarity, can be deduced analytically assuming a pure axial dipole at the centre of the Earth in order to obtain self-similar conditions while decreasing the Earth's magnetic field intensity.

The polar cap latitude can be obtained analytically with the superposition method considering a pure axial dipole (Hill \& Rassbach, 1975). In this case, from eq. A3 of Hill \& Rassbach (1975) $\cos ^{2}\left(\lambda_{\mathrm{P}}\right)=3\left(R_{\mathrm{T}} / R_{\mathrm{MP}}\right) /\left[2+\left(R_{\mathrm{T}} / R_{\mathrm{MP}}\right)^{3}\right]$. Since $\left(R_{\mathrm{T}} / R_{\mathrm{MP}}\right)^{3} \ll 1$, then $\cos \left(\lambda_{\mathrm{P}}\right) \propto R_{\mathrm{MP}}{ }^{-1 / 2}$ and consequently $\cos \left(\lambda_{\mathrm{P}}\right) \propto M^{-1 / 6}$. In this case, $\lambda_{\mathrm{P}}=68.1^{\circ}$ for $M=8.10^{22} \mathrm{Am}^{2}$ and $|\mathbf{B}|=49 \mathrm{nT}$. This scaling law is equal to that obtained by Siscoe \& Chen (1975) where they equate the magnetic flux at the polar cap to the flux through the magnetosphere tail.

The polar cap area, $A_{\mathrm{P}}=2 \pi R_{\mathrm{T}}{ }^{2}\left[1-\cos \left(90-\lambda_{\mathrm{P}}\right)\right]$ (from a spherical cap area equation), varies more strongly with $M$. This follows from considering $\cos \left(90-\lambda_{\mathrm{P}}\right)=\left[1-\cos \left(\lambda_{\mathrm{P}}\right)\right]^{1 / 2}$, which after a Taylor series approximation results in $A_{\mathrm{P}} \propto M^{-1 / 3}$. Then, a 50 per cent decrease in $M$, with $M=8.10^{22} \mathrm{Am}^{2}$ and $|\mathbf{B}|=49 \mathrm{nT}$ at $R_{\mathrm{MP}}$, gives a small $\lambda_{\mathrm{P}}$ shift from $68.1^{\circ}$ to $65.3^{\circ}$, with a consequent $\sim 27$ per cent increase 


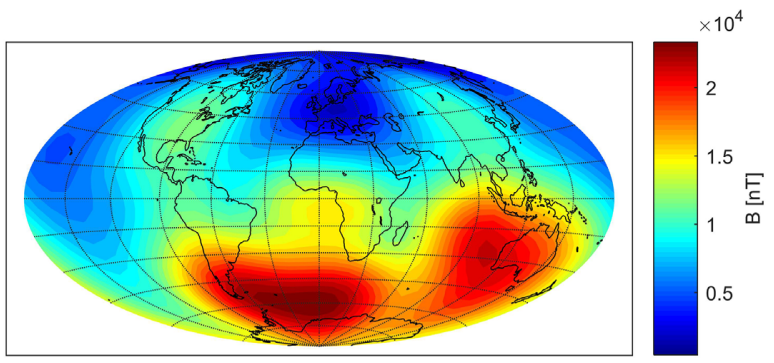

(a)

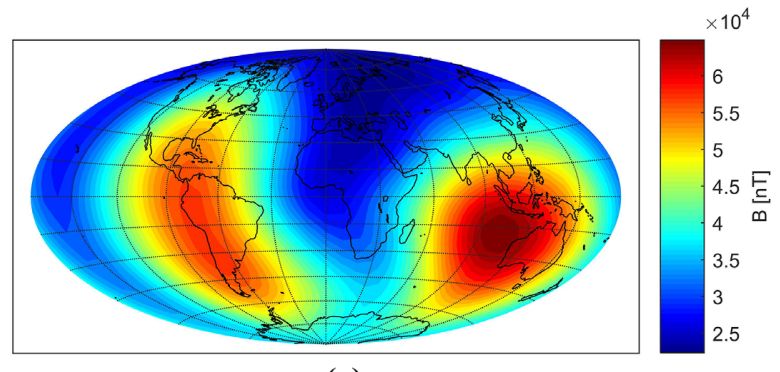

(c)

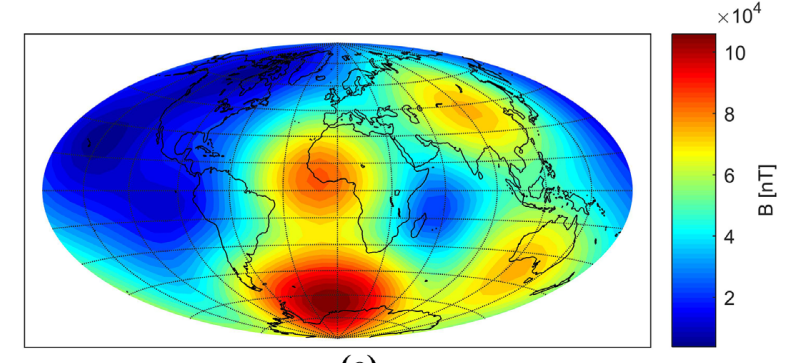

(e)

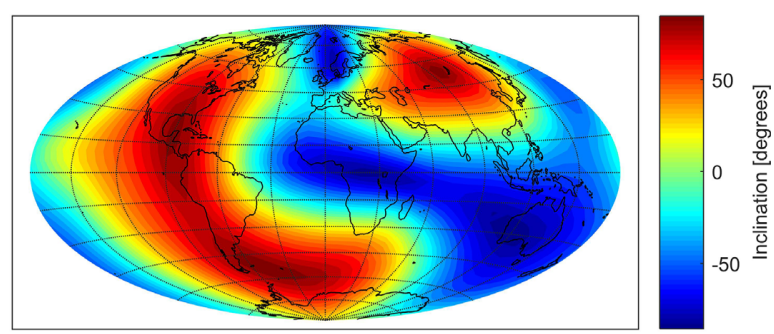

(b)

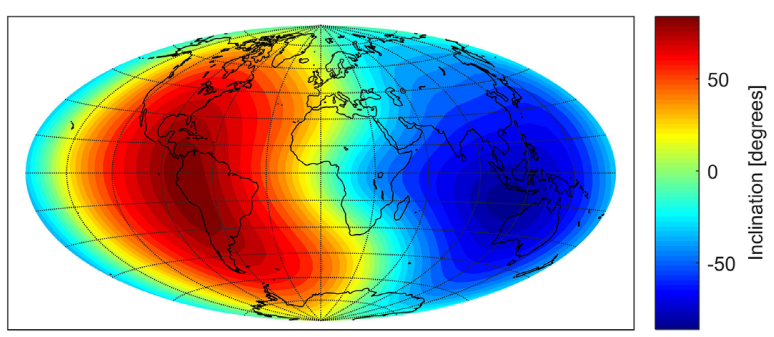

(d)

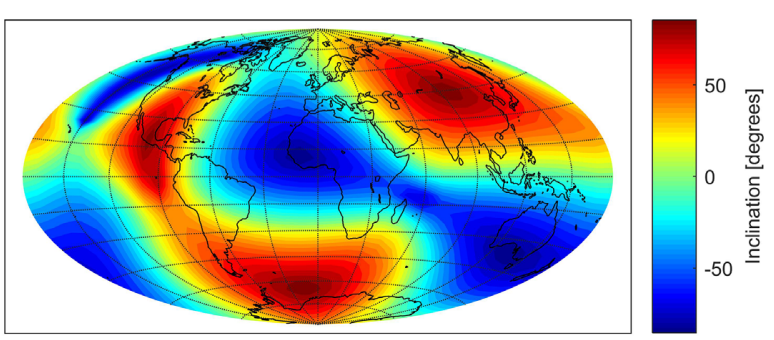

(f)

Figure 2. As in Fig. 1 for (a and b) axial dipole collapse, (c and d) dipole rotation where axial dipole energy is transferred to the equatorial dipole and (e and f) energy cascade where dipolar energy is transferred to the quadrupolar and octupolar terms. Note the different scales for intensity.

in the polar cap area. A 90 per cent decrease in $M$ would result in a $\lambda_{\mathrm{P}}$ shift to $56.9^{\circ}(\sim 16$ per cent decrease $)$ and an almost double polar cap area. These scaling laws predict that dipole decrease will be accompanied by a shift to lower latitudes of the polar caps' limit but at a rate much lower than the relative dipole moment decrease.

In general, predictions from the analytical scaling laws for a pure axial dipole are not valid for more complex scenarios. In the cases analysed here, where multipolar components are included, as the axial dipole decreases the self-similarity assumption becomes less valid. Polar cap shapes are no longer circles described by single latitudes delineating its limits. Instead, they elongate and their centres move towards lower latitudes, eventually being more than two for a dipole collapse.

\section{CONCLUDING REMARKS}

Using eq. (6) to obtain the magnetopause subsolar distance it can be noted that, although the most robust and dramatic feature of reversals is the decrease in the axial dipole, even for a dipole moment decrease to 10 per cent of its present value the magnetopause stand-off distance would be at $\sim 5$ Earth radii. For a given quadrupole/dipole ratio at the Earth's surface, at the magnetopause the ratio would decrease by a factor 5 because the quadrupolar component decreases with $1 / r^{4}$ whereas the dipole decreases with $1 / r^{3}$. The dipole field is therefore influential in determining the polar caps locations even when it is strongly reduced.
Westward drift of non-dipole components (e.g. Campbell 2003; Aubert et al. 2013) were not considered in this study, so polar caps shown in the simulated scenarios could probably appear in different longitudes. Our results are therefore robust in terms of the number of polar caps and their latitudes, whereas their longitudes may drift or oscillate about a preferred location. Such a motion may be confined to a limited longitudinal range if strong lower mantle thermal heterogeneity controls the geodynamo (e.g. Amit et al. 2015).

Our results provide a glimpse into possible magnetospheric conditions during reversals. The superposition of the interplanetary field with a dominantly axial dipole field during chrons yields at the Earth's surface two poles near the dipole axis (Fig. 3a), whereas during reversals either two poles near the equator (Fig. 3b) or a more complex morphology of more than two poles (Figs $3 \mathrm{c}-\mathrm{e}$ ) may emerge. The superposition of the internal and external field may give strong longitude dependence of the magnetospheric conditions during reversals (Figs 3b-e). This result demonstrates the likely complexity of the field morphology in the magnetosphere when the internal core origin geomagnetic field undergoes dramatic changes such as polarity transitions.

The locations of polar caps exhibit dramatic variability for different reversal scenarios. We found that polar caps typically appear where both the field intensity and the absolute value of its inclination are large. Two polar caps near the dipole axis intersection with the Earth's surface prevail for a dipole decrease to a certain extent 


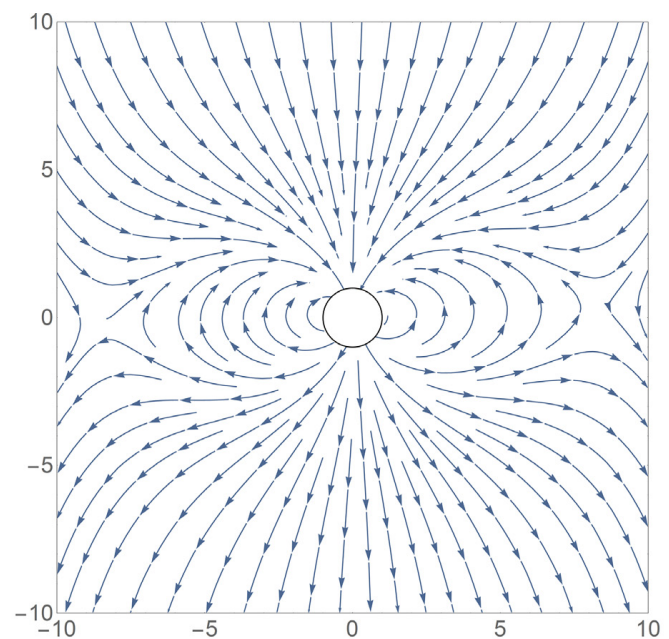

(a)

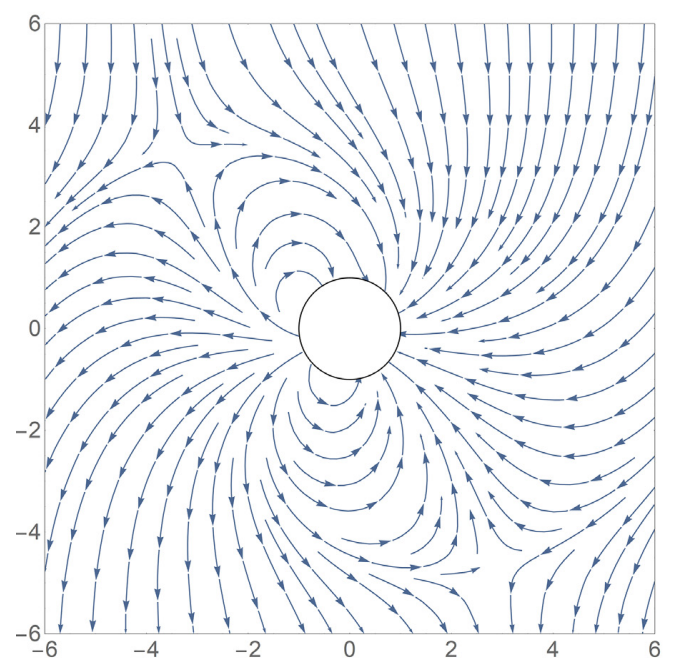

(b)

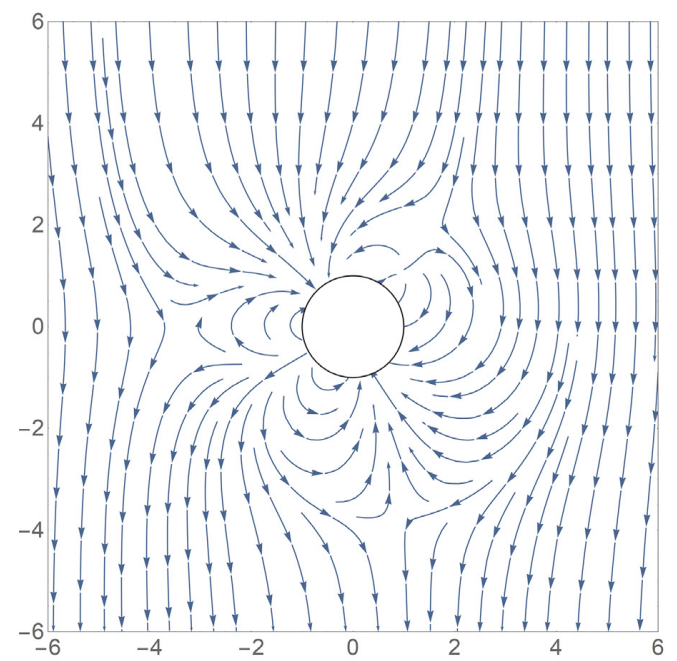

(d)

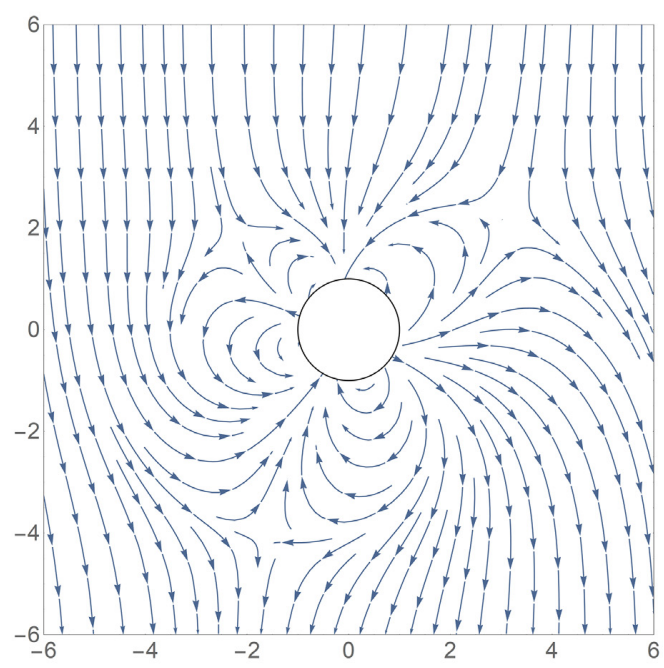

(c)

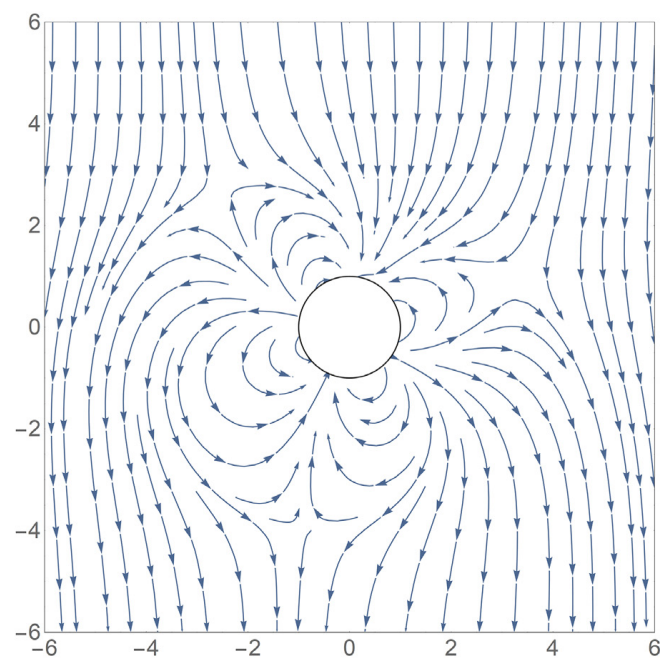

(e)

Figure 3. Superposition of a uniform external field and the Earth's internal magnetic field (blue arrows) for (a) present field configuration (prime meridian cross-section), (b) axial dipole collapse $\left(90^{\circ}\right.$ meridian cross-section), (c) axial dipole collapse (prime meridian cross-section), (d) energy cascade $\left(90^{\circ}\right.$ meridian cross-section) and (e) energy cascade (prime meridian cross-section). Inner circle represents the Earth. Note the different scales of panel (a). 


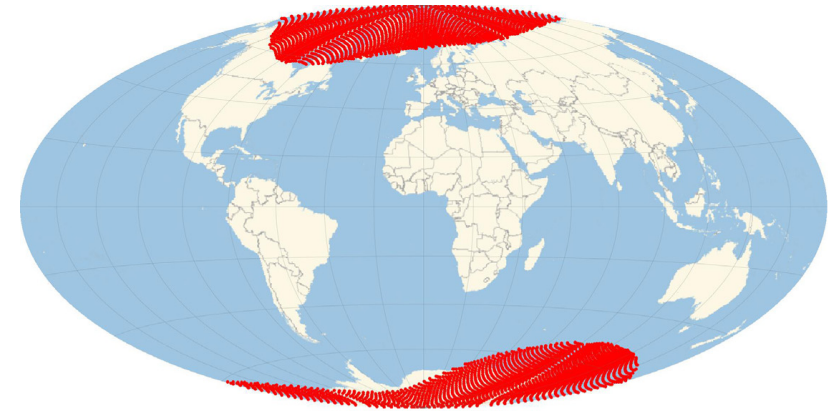

(a)

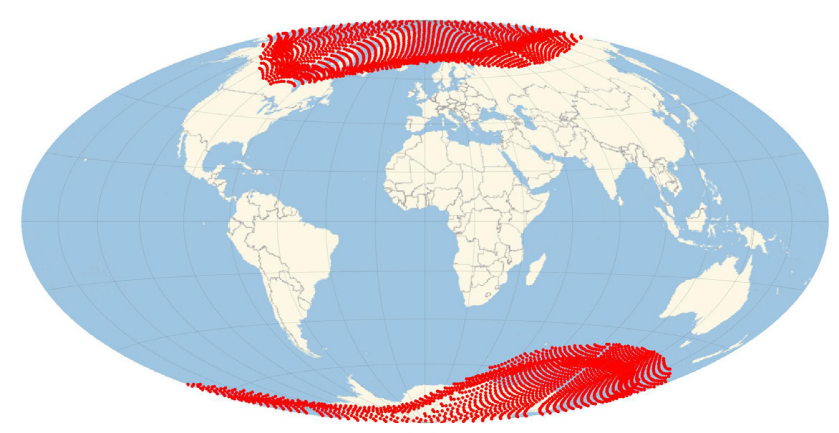

(b)

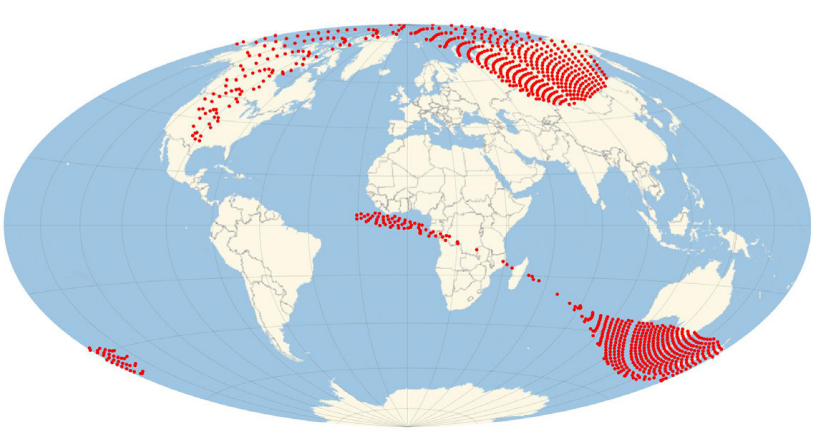

(c)

Figure 4. Polar caps' locations for (a) present field configuration, (b) 50 per cent reduction of the dipolar components and (c) 90 per cent reduction of the dipolar components.

(Figs $4 \mathrm{a}$ and $\mathrm{b}$ ). However, when the dipole is further reduced, nontrivially the northern polar cap remains at high latitudes whereas the southern polar cap moves to mid-latitudes including a long thin branch all the way to the equator (Fig. 4c). A complete axial dipole collapse gives a pair of polar caps, both at mid-latitudes of the southern hemisphere (Fig. 5a). These results point to a remarkable hemispheric dichotomy, which is most likely related to the more non-dipolar field below the southern hemisphere, in particular the presence of reversed flux patches below the South Atlantic on the core-mantle boundary (e.g. Terra-Nova et al. 2017). Finally, if reversals occur due to an energy cascade from the dipole to higher degrees, more than two polar caps may appear. In our energy cascade scenario, four polar caps at various latitudes of both hemispheres prevail (Fig. 5c). These results indicate that polar caps may reach extraordinary locations during reversals.

The magnetospheric field configuration and the resulting shape of the polar cap region are much more complex than described here. The real interaction of the solar wind and the Earth's magnetic field, together with coupling mechanisms, feedbacks and the impact of

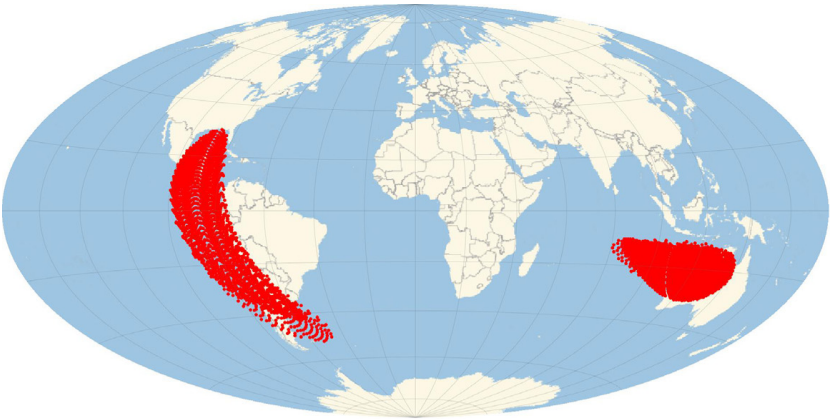

(a)

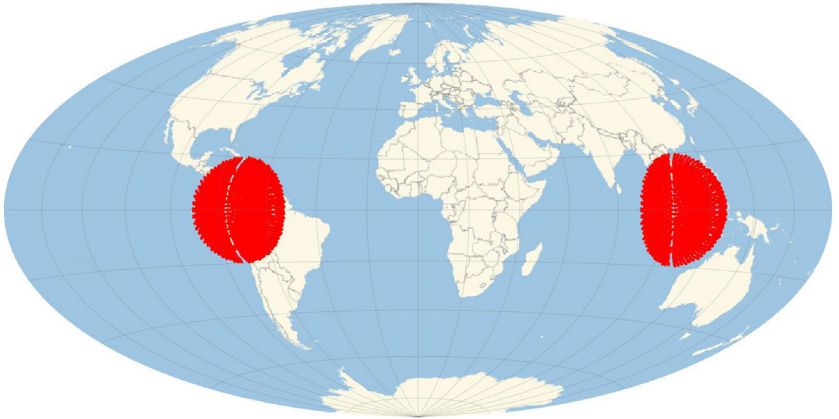

(b)

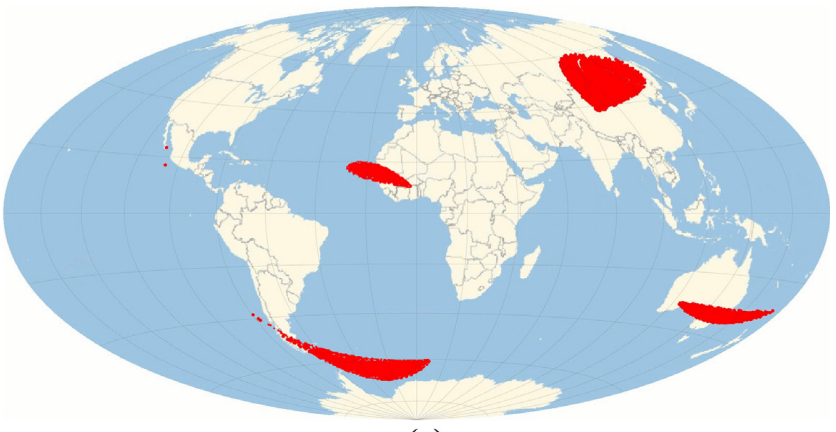

(c)

Figure 5. As in Fig. 4 for (a) axial dipole collapse, (b) dipole rotation where axial dipole energy is transferred to the equatorial dipole and (c) energy cascade where dipolar energy is transferred to the quadrupolar and octupolar terms.

the ionosphere, are not included in our model. However, because polar caps are assessed at the Earth's surface, the main factor determining the polar caps' locations is the internal geomagnetic field. We therefore consider that, overall, for the geomagnetic field scenarios considered here, the real polar cap configurations would not be significantly different than in our results.

Stadelman et al. (2010), who also did not use an MHD model, traced particle trajectories in palaeomagnetospheres under conditions of a geomagnetic polarity transition using quantitative parametric models, and assuming a fixed-shape magnetopause given by a half-sphere with radius $r_{\mathrm{m}}$ representing the dayside and a semiinfinite cylinder with radius $r_{\mathrm{m}}$ on the nightside, with the Earth placed within the half-sphere. They argued that MHD simulations are computationally more expensive and their spatial resolution is limited in some regions to treat trajectories. They scaled the size of this fixed-shape magnetosphere according to the magnetic moment of each considered configuration. In this way they estimated cut-off latitudes, which divide regions with and without particle impacts and impact areas by following the trajectories of particles with a 
range of energies initiating outside the magnetosphere, similar to our procedure of following open magnetic lines to the Earth. They considered 'closed' magnetospheres, and 'inner' lines were reached according to the particle's energy. This is equivalent to open lines in our case. Their impact areas are 'equivalent' to polar caps for certain particles energy. For the axial dipole, in fact, their impact area corresponds to the region between $90^{\circ}$ and a cut-off latitude, which would represent our polar cap limit. The increasing particle energies that increase the impact area would correspond in our case to a decreasing magnetic field (which they also considered). Even though Stadelman et al. (2010) considered only dipole and quadrupole moments they give a generalization to higher multipoles.

Since polar cap boundaries correspond to the inner limit of the auroral oval, which becomes enlarged under geomagnetic storm conditions, increasing polar cap zones could result in visible auroras in various regions of the Earth. Although our results do not point to a significant increase in polar caps' area during reversals, some reversal scenarios may dramatically decrease the latitudes of polar caps (Fig. 5), potentially reaching highly populated areas. This might have harmful consequences since these are zones vulnerable to particle precipitation effects. Maybe the most relevant problem in these areas is the total 'blackout' of ionospherically propagating radio signals affecting long-distance communications as well as satellite geolocation, leaving airplanes and ships temporarily without positioning systems, with interruptions that can last several days. Another consequence of particle precipitation is changes in atmosphere chemistry, with dissociation of ozone maybe the worst of these alterations (Winkler et al. 2008; Glassmeier \& Vogt 2010; Sinnhuber et al. 2012). Understanding the dependence of polar caps' locations on drastic geomagnetic field changes such as reversals, but also due to dipole decrease as has been observed during the historical era (e.g. Finlay 2008), may provide both fundamental understanding to the interaction of the solar wind with the geomagnetic field as well as practical help to society in dealing with technological challenges.

\section{ACKNOWLEDGEMENTS}

We thank Prof. Karl-Heinz Glassmeier and an anonymous reviewer for their constructive and useful comments that have improved this manuscript. We thank ISSI (International Space Science Institute, Bern) and ISSI-BJ (International Space Science Institute, Beijing) for their hospitality and support of the international team on 'Climate Change in the Upper Atmosphere' where many ideas in this work were discussed. This work was supported by Projects PIUNT E642 and PICT 2015-0511.

The model and simulations are available from Bruno S. Zossi upon request (brunozossi@hotmail.com).

\section{References}

Akasofu, S.I., 1983. Evolution of ideas in solar-terrestrial physics, Geophys. J. R. astr. Soc., 74, 257-299.

Amit, H. \& Olson, P., 2010. A dynamo cascade interpretation of the geomagnetic dipole decrease, Geophys. J. Int., 181, 1411-1427.

Amit, H., Leonhardt, R. \& Wicht, J., 2010. Polarity reversals from paleomagnetic observations and numerical dynamos simulations, Space Sci. Rev., 155, 293-335.

Amit, H., Choblet, G., Olson, P., Monteux, J., Deschamps, F., Langlais, B. \& Tobie, G., 2015. Towards more realistic core-mantle boundary heat flux patterns: a source of diversity in planetary dynamos, Prog. Earth Planet. Sci., 2, 2-26.
Aubert, J., Aurnou, J. \& Wicht, J., 2008. The magnetic structure of convective-driven numerical dynamos, Geophys. J. Int., 179, 945-956.

Aubert, J., Finlay, C.C. \& Fournier, A., 2013. Bottom-up control of geomagnetic secular variation by the Earth's inner core, Nature, 502, 219-223.

Beard, D.B., 1960. Interaction of the solar plasma with the Earth's magnetic field, Phys. Rev. Lett., 5, 89-91.

Campbell, W.H., 2003. Introduction to Geomagnetic Fields, Cambridge Univ. Press.

Clement, B.M., 2004. Dependence of the duration of geomagnetic polarity reversals on site latitude, Nature, 428, 637-640.

Feldstein, Y.I., 2016. The discovery and the first studies of the auroral oval: a review, Geomagn. Aeron., 56, 129-142.

Finlay, C.C., 2008. Historical variation of the geomagnetic axial dipole, Phys. Earth planet. Inter., 170, 1-14.

Glassmeier, K.H. \& Vogt, J., 2010. Magnetic polarity transitions and biospheric effects, Space Sci. Rev., 155, 1-4.

Glassmeier, K.H., Vogt, J., Stadelmann, A. \& Buchert, S., 2004. Concerning long-term geomagnetic variations and space climatology, Ann. Geophys., 22, 3669-3677.

Gombosi, T. et al., 2004. Solution-adaptive magnetohydrodynamics for space plasmas: Sun-to-Earth simulations, Comput. Sci. Eng., 6, 14-35.

Hastings, C., Mischo, K. \& Morrison, M., 2016. Hands-on Start to Wolfram Mathematica and Programming with the Wolfram Language, 2nd edn, Wolfram Media, Inc.

Hill, T.W. \& Rassbach, M.E., 1975. Interplanetary magnetic field direction and the configuration of the day side magnetosphere, J. geophys. Res., $8 \mathbf{8 0}$ $1-6$.

Huguet, L. \& Amit, H., 2012. Magnetic energy transfer at the top of Earth's core, Geophys. J. Int., 190, 856-870.

Hulot, G., Lhuillier, F. \& Aubert, J., 2010. Earth's dynamo limit of predictability, Geophys. Res. Lett., 37.

Hunsucker, R.D. \& Hargreaves, J.K., 2003. The High-Latitude Ionosphere and its Effects on Radio Propagation, Cambridge Univ. Press.

Jacobs, J.A., 1994. Reversals of the Earth's Magnetic Field, Cambridge Univ. Press.

Lowes, F.J., 1974. Spatial power spectrum of the main geomagnetic field, and extrapolation to the core, Geophys. J. R. astr. Soc., 36, $717-730$.

Merrill, R.T., McElhinny, M.W. \& McFadden, P.L., 1998. The Magnetic Field of the Earth, Academic Press.

Morzfeld, M., Fournier, A. \& Hulot, G., 2017. Coarse predictions of dipole reversals by low-dimensional modeling and data assimilation, Phys. Earth planet. Inter, 262, 8-27.

Polvani, L.M., Clement, A.C., Medeiros, B., Benedict, J.J. \& Simpson, I.R., 2017. When less is more: opening the door to simpler climate models, EOS Earth Space Sci. News, 98.

Russell, C.T., 2000. The polar cusp, Adv. Space Res., 25, 1413-1424.

Sinnhuber, M., Nieder, H. \& Wieters, N., 2012. Energetic particle precipitation and the chemistry of the Mesosphere/Lower thermosphere, Surv. Geophys., 33(6), 1281-1334.

Siscoe, G.L. \& Chen, C.K., 1975. The paleomagnetosphere, J. geophys. Res., 80, 4575-4680.

Siscoe, G.L. \& Sibeck, D.G., 1980. Effects of nondipole components on auroral zone configurations during weak dipole field epochs, J. geophys. Res., 85, 3549-3556.

Stadelman, A., Vogt, J., Glassmeier, K.H., Kallenrode, M.B. \& Voigt, G.H., 2010. Cosmic ray and solar energetic particle flux in paleomagnetospheres, Earth Planets Space, 62, 333-345.

Sterenborg, M.G., Cohen, O., Drake, J.J. \& Gombosi, T.I., 2011. Modeling the young Sun's solar wind and its interaction with Earth's paleomagnetosphere, J. geophys. Res., 116.

Stern, D.P., 1973. A study of the electric field in an open magnetospheric model, J. geophys. Res., 78, 7292-7305.

Terra-Nova, F., Amit, H., Hartmann, G.A., Trindade, R.I.F. \& Pinheiro, K.J., 2017. Relating the South Atlantic Anomaly and geomagnetic flux patches, Phys. Earth planet. Inter., 266, 39-53. 
Thébault, E. et al., 2015. International Geomagnetic Reference Field: the 12th generation, Earth Planets Space, 67.

Valet, J.P. \& Fournier, A., 2016. Deciphering records of geomagnetic reversals, Rev. Geophys., 54, 410-446.

Valet, J.P., Fournier, A., Courtillot, V. \& Herrero-Bervera, E., 2012. Dynamical similarity of geomagnetic field reversals, Nature, 490, 89-93.

Vogt, J. \& Glassmeier, K.H., 2000. On the location of trapped particle populations in quadrupole magnetospheres, J. geophys. Res., 105, 13063 13071.

Vogt, J. \& Glassmeier, K.H., 2001. Modelling the paleomagnetosphere: strategy and first results, Adv. Space Res., 28, 863-868.

Vogt, J., Zieger, B., Glassmeier, K.H., Stadelmann, A., Kallenrode, M.B., Sinnhuber, M. \& Winkler, H., 2007. Energetic particles in the paleomagnetosphere: reduced dipole configurations and quadrupolar contributions, J. geophys. Res., 112.
Willis, D.M., Holder, A.C. \& Davis, C.J., 2000. Possible configurations of the magnetic field in the outer magnetosphere during geomagnetic polarity reversals, Ann. Geophys., 18, 11-27.

Winkler, H. et al., 2008. Modeling impacts of geomagnetic field variations on middle atmospheric ozone responses to solar proton events on long timescales, J. geophys. Res., 113.

Zieger, B., Vogt, J., Glassmeier, K.H. \& Gombosi, T.I., 2004. Magnetohydrodynamic simulation of an equatorial dipolar paleomagnetosphere, $J$. geophys. Res., 109.

Zieger, B., Vogt, J. \& Glassmeier, K.H., 2006. Scaling relations in the paleomagnetosphere derived from MHD simulations, J. geophys. Res., 111

Zossi, B.S., Elias, A.G. \& Fagre, M., 2018. Ionospheric conductance spatial distribution during geomagnetic field reversals, J. geophys. Res., 123, 2379-2397. 